\title{
Comprehensive medication reviews by ward- based pharmacists in Swedish hospitals: What does the patient have to say?
}

\author{
KEMPEN, T.G.H., KÄLVEMARK, A., GILLESPIE, U., STEWART, D.
}

This is the peer reviewed version of the following article: KEMPEN, T.G.H., KÄLVEMARK, A., GILLESPIE, U. and STEWART, D. 2020. Comprehensive medication reviews by ward-based pharmacists in Swedish hospitals: what does the patient have to say? Journal of evaluation in clinical practice [online], 26(1), pages 149-157, which has been published in final form at https://doi.org/10.1111/jep.13121. This article may be used for noncommercial purposes in accordance with Wiley Terms and Conditions for Use of Self-Archived Versions. 
Title: Comprehensive medication reviews by ward-based pharmacists in Swedish hospitals: what does the patient have to say?

Authors and affiliations: Thomas G.H. Kempen ${ }^{\text {ab* }}$, Amanda Kälvemark ${ }^{\mathrm{a}}$, Ulrika Gillespie ${ }^{\mathrm{a}}$ and Derek Stewart ${ }^{\mathrm{cd}}$ ${ }^{a}$ Department of Medical Sciences, Uppsala University, Uppsala, Sweden; ${ }^{b}$ Hospital Pharmacy Department, Uppsala University Hospital, Uppsala, Sweden; ' School of Pharmacy and Life Sciences, Robert Gordon University, Aberdeen, Scotland; ${ }^{\mathrm{d}}$ College of Pharmacy, Qatar University, Doha, Qatar

* Corresponding author at; Postal address: Akademiska sjukhuset, klinikapotekare, ingång 226, 75185 Uppsala, Sweden; Telephone number: +46(0)18-611 16 40. E-mail address: thomas.kempen@medsci.uu.se

\begin{abstract}
Rationale, aims, and objectives: Inappropriate medication prescribing and use among older patients is a major patient safety and healthcare problem. To promote appropriate medication prescribing and use, comprehensive medication reviews (CMRs) by ward-based pharmacists, including follow-up telephone calls after hospital discharge, have been conducted in older patients in the context of a randomised controlled trial (RCT). One of the key actors in a CMR is the patient. To support the understanding of the effects of CMRs on patients' health outcomes and improve clinical practice, knowledge about the patient perspective is needed. We therefore aimed to explore older patients' experiences with, and views on, hospital-initiated CMRs and follow-up telephone calls by ward-based clinical pharmacists within an RCT.

Methods: We conducted in-depth semi-structured interviews with fifteen patients (66-94 years) and carers from four hospitals in Sweden. Discussion topics included communication, information, decision-making, and effects on the patient. Interviews took place after discharge, were audio-recorded, transcribed verbatim, and thematically analysed using a framework approach.

Results: In general, patients' experiences and views were positive. Seven key themes were identified: 1 ) feeling of being taken care of and heterogenous health effects; 2 ) the pharmacist is competent; 3 ) despite the unclear role of pharmacists, their involvement is appreciated; 4) patients rely on healthcare professionals for decision-making; 5) importance of being informed, but receiving and retaining information is problematic; 6) time, location and other factors influencing the effectiveness of CMRs; 7) generic substitution is a problem. Conclusions: Older patients generally have positive experiences with and views on CMRs and follow-up telephone calls. However, some factors, like the unclear role of the ward-based pharmacist and problems with receiving and retaining information, may negatively impact the effectiveness of these interventions. Future initiatives on hospital-initiated CMRs by clinical pharmacists should address these negative factors and utilize the positive views.
\end{abstract}

\title{
Keywords
}

Interdisciplinary Health Team; Patient Involvement; Health Services for the Aged; Qualitative Research; Review, Drug Utilization; Clinical Pharmacists 


\section{Introduction}

Inappropriate medication prescribing and use among older patients is a major patient safety and healthcare problem of global significance [1,2]. Up to $30 \%$ of all hospitalisations in older patients are medication-related and half of these are potentially preventable [3-5]. Even during hospitalisation and after discharge, many problems with the management of medication exist $[6,7]$. Performing comprehensive medication reviews (CMRs), a structured, critical examination of a patient's medications with the objective of reaching an agreement with the patient about treatment [8], in older patients can improve medication prescribing, optimise treatment benefit and minimise harm $[9,10]$. Clinical pharmacists have been introduced to multiprofessional teams at ward level to perform CMRs in hospitalised patients [10]. However, a lack of high quality evidence inhibits widespread implementation [11].

To fulfil the need for evidence, a cluster-randomised controlled trial, Medication Reviews Bridging Healthcare (MedBridge; www.clinicaltrials.gov: NCT02986425), is currently being performed in Swedish hospitals [12]. This trial aims to study the effects of CMRs, including follow-up after hospital discharge, on older patients' healthcare utilisation compared to usual care, i.e. no CMR. Intervention patients may receive a CMR in which a ward-based clinical pharmacist collaborates with the ward physician and the patient or carer (intervention 1), or two follow-up telephone calls after hospital discharge in addition to a CMR (intervention 2), see Box 1. In both interventions, patient or carer involvement is an important element.

Box 1

Study interventions within the Medication Reviews Bridging Healthcare (MedBridge) trial [12]

\section{Study interventions within the MedBridge trial}

\section{Intervention 1 'Comprehensive medication review' (CMR)}

A clinical pharmacist collaborates with the ward physician and the patient or carer to improve the appropriateness and effectiveness of the prescribed medications and their use, and to minimise the risk of medication-related problems. As soon as possible after admission, preferably within $24 \mathrm{~h}$, a clinical pharmacist interviews the patient or carer interview to ensure the hospital list of medications is consistent with what medications the patient used at home, and to identify any medicationrelated problems experienced by the patient. The clinical pharmacist then performs, in collaboration with the ward physician, a structured, critical examination of all the patient's medications in relation to the patient's conditions, based on information from the patient and the medical record. The objective is to reach an agreement between the pharmacist, physician and patient (or carer) about the continued appropriateness and effectiveness of the treatment, optimising the impact of medications and minimising the number of medication-related problems. Other issues, such as adherence, practical use of the medications (e.g. how to use an inhaler), dosage forms, adverse effects, interactions, and the patient's understanding of the condition and its treatment are considered when appropriate. The effects of medication changes are monitored during hospital stay and a final check of the patient's medication list and prescriptions is performed by the clinical pharmacist before discharge. If deemed necessary, additional contact between the physician, pharmacist and/or patient may take place during the admission to optimise medication treatment.

\section{Intervention 2 'CMR with follow-up after discharge'}

The same as intervention 1, with the addition of the pharmacist having the possibility to send an electronic medication referral to the patients' general practitioner with recommendations on treatment monitoring or follow-up actions. Next to that, two follow-up telephone calls to the patient are made by the pharmacist 2-7 days and 1-2 months after discharge. The goal of these calls is to ensure that all information has been understood correctly and to find out if any problems, concerns or questions have arisen after hospital stay. The second phone call also aims to increase treatment adherence. If necessary, the clinical pharmacist provides counselling or takes any other additional actions.

\section{Control 'Usual care'}

Usual hospital care without a clinical pharmacist involved.

For a clinical trial in which the intervention comprises of multiple components and is dependent on the interaction with its context and behaviour of people, it is highly recommended to perform a process evaluation alongside the trial [13]. Qualitative approaches, such as interviews, focus groups and researcher observations are often used within process evaluations to provide detailed information about the implementation, mechanism of impact and the context of the different intervention components [13]. One of the key actors in a CMR is the patient. Positive patient experience is associated with clinical effectiveness and patient safety [14]. Several quantitative tools and frameworks, such as the Picker Patient Experience Questionnaire, have been developed to measure patient experience [15]. Survey studies 
generally report positive experience and satisfaction with CMRs [16-18], but a qualitative approach providing in-depth data is needed to get an understanding of the factors influencing these experiences in order to improve clinical practice [19].

Although systematic reviews on qualitative studies investigating patient experience with different healthcare interventions exist $[20,21]$, few qualitative studies have evaluated this regarding hospital-initiated CMRs, with or without follow-up calls, or similar services involving clinical pharmacists [22,23]. Garcia et al. [22] explored the experiences of coronary heart disease patients with pharmacist-led follow-up, including medication review, upon and after discharge from hospital in Norway. Patients expressed increased medication knowledge, and they felt safer, reassured and more involved in the management of their medication. The importance of physician-pharmacist collaboration was emphasized, but the role of pharmacists needed to be clarified in order for patients to know what to expect from them [22]. Similar findings were reported by Morecroft et al. [23]: older patients who had received hospital-based pharmacy services in the United Kingdom responded positively to the pharmacist's availability, communication skills and information provided. However, limited knowledge of the pharmacists' role was an impeding factor to constructive interaction between patients and pharmacists [23].

The extent of transferability of these findings to other contexts, such as the Swedish hospital setting, is unclear. A detailed exploration of the patients' perspective can support the understanding of the effects of hospital-initiated CMRs on patients' health outcomes. We therefore present this nested qualitative study which aimed to explore older patients' experiences with and views on hospital-initiated CMRs and follow-up telephone calls by ward-based clinical pharmacists within the MedBridge trial.

\section{Methods}

\subsection{Study design and methodological approach}

A qualitative design with in-depth interviews was chosen to gain rich accounts of patient experiences and views. We have taken an interpretive approach as proposed by Ritchie and Lewis [24]. That is, we accepted that the reality exists independently of individual subjective understanding, but that it is only accessible to the researchers via the participants' interpretations. The Standards for reporting qualitative research (SRQR) [25] and Consolidated criteria for reporting qualitative research (COREQ) [26] were used to design and report the study.

\subsection{Setting}

This was a nested study within the MedBridge trial which was simultaneously being performed at four hospitals in three regions in Sweden: Uppsala University Hospital and the hospitals of Enköping, Västerås and Gävle. The hospitals were chosen because of the presence of wards where physicians and nurses are supported by a clinical pharmacist. The pharmacists had either completed a full-time one-year postgraduate programme in clinical pharmacy, in which the performance of CMRs played a central role, or they had completed undergraduate courses in clinical pharmacy and advanced pharmacotherapy. All pharmacists also had at least six months working experience with performing CMRs in a multi-professional team. Two wards per hospital were included. The wards differed in terms of medical specialty: geriatric, internal medicine, stroke, diabetes and nephrology. All patients aged 65 or older who were admitted to one of the wards were asked for informed consent to participate in the MedBridge trial [12]. Patients were excluded if they were in a palliative stage, had been subject to a medication review within the last 30 days, resided in another than the hospital's region, or were admitted for only one day. During admission, patients received either one of the study interventions, as described in Box 1 , or usual care without a clinical pharmacist involved at the ward. In total, 2640 patients (intervention 1: 922, intervention 2: 824, control: 894) were included in the MedBridge trial between February 2017 and November 2018.

\subsection{Sampling and recruitment}

The potential participants in this study were participants within the MedBridge trial who had received one of the two interventions and were taking care of their own medication management in the ambulant setting 
according to information from their medical record. Spouse or children responsible for the patient's medication management (carers) were also considered potential participants. Patients were excluded if they could not specifically remember the contact with the clinical pharmacist during hospital stay.

Purposive sampling was used to be able to identify themes which cut across a variety of patients [24]. In this approach, patients were sampled to ensure coverage across hospital wards and heterogeneity in terms of age, gender, intervention ( 1 or 2 ), clinical pharmacist involved, and the number (high or low) of medicationrelated problems identified during the CMR. Those selected for interview were invited by telephone. The interview was preferably planned approximately one to four weeks after the patient's hospital discharge to minimise problems with recalling the experience. For intervention 2 patients, the interview would be after the first follow-up telephone call, but before the second call. Recruitment and interviewing took place between September 2017 and April 2018. Participants provided written informed consent before the start of the interview. The researchers had a pharmacy (TK) and nursing (AK) background, were involved as study coordinators within the MedBridge trial, and had received training in qualitative research and interviewing prior to the study. The researchers had not been in contact with the participants prior to recruitment. Regular meetings were held between the researchers to discuss the recruitment process and ensure heterogeneity in the sample.

Sampling was continued to the point of data saturation, defined as the point when no new additional data are found that develop a conceptual category or theme [27]. In our approach, 12 initial interviews were conducted and analysed, to identify key, recurring themes. After 12 interviews, three consecutive interviews with no new themes emerging were needed to reach the point of data saturation [27].

\subsection{Data generation and analysis}

A semi-structured discussion guide was used to guide the interviews (Appendix A in Supplementary material). The content of the discussion guide was based on the topics within the NHS Patient Experience Framework [28], the Picker Patient Experience Questionnaire [15], and the different intervention components within the MedBridge trial [12]: communication with the clinical pharmacist during hospital stay; decision-making process within the CMR; information provided during hospital admission; two telephone calls by the clinical pharmacist (for intervention 2 patients only); overall effect of the intervention on the patient. One researcher (TK) conducted all interviews at the participants' hospital or residence. The participants were encouraged to be frank and speak freely. The interviewer asked follow-up questions rooted in the main topics to elaborate, clarify and confirm understanding. The interviews were audio-recorded, transcribed verbatim by one researcher (AK) and checked for transcribing accuracy by the same researcher who performed the interviews. Data analysis followed a framework approach as proposed by Ritchie and Spencer [24]. This approach involves familiarisation with the data, identifying themes, coding, constructing and filling the framework and interpretation. The two researchers discussed the coding framework being used and coded each interview independently using Open Code 4.03 [29], with consensus reached by discussion. Swedish was the primary language for the interviews, transcripts and coding. In the final interpretation phase, the sub-themes and key themes were translated into English. An audit trail with all research steps taken was kept throughout the recruitment, data generation and analysis process.

\subsection{Ethics and research trustworthiness}

The MedBridge trial received ethical approval from the Swedish Central Ethical Review Board (CEPN; registration number: Ö 21-2016) and additional ethical approval from the Regional Ethical Review Board in Uppsala for the patient interviews (EPN Uppsala; registration number: 2016-251-1). Research trustworthiness and rigour [30] were addressed in diverse ways throughout the research process (Table 1).

Table 1 Strategies to ensure trustworthiness and rigour of the research process

\begin{tabular}{|l|l|}
\hline Credibility & $\begin{array}{l}\text { - Adopted appropriate, well recognised research methods [24,27] } \\
\text { - Familiarity among the researchers with the study context (2.3. Sampling and recruitment) } \\
\text { - Description of background and training of the researchers (2.3. Sampling and recruitment) }\end{array}$ \\
\hline & $\begin{array}{l}\text { - Triangulation via use of different types of participants (2.3. Sampling and recruitment) } \\
\text { - Encouragement of participants to be frank and talk freely (2.4. Data generation and analysis) } \\
\text { - Scrutiny of the project by an experienced, external researcher (DS) }\end{array}$ \\
\hline
\end{tabular}




\begin{tabular}{|l|l|}
\hline & - Examination of previous research to frame findings (4.1. Discussion) \\
\hline Transferability & - Description of the context (2.2. Setting) and comprehensive description of the findings (3. Results) \\
\hline Dependability & $\begin{array}{l}\text { - Comprehensive methodological description to allow the study to be repeated (2. Methods) } \\
\text { - Reflective appraisal of the study (4.1. Discussion) }\end{array}$ \\
\hline Confirmability & $\begin{array}{l}\text { - Description of the researchers' ontological and epistemological position (2.1. Study design and } \\
\text { methodological approach) } \\
\text { - Recognition of limitations in study's methods and their potential effects (4.1. Discussion) } \\
\text { - Audit trail of the recruitment, data generation and analysis (2.4. Data generation and analysis) }\end{array}$ \\
\hline
\end{tabular}

\section{Results}

\subsection{Patient characteristics}

In total, 15 interviews were conducted out of 36 patients or carers that were approached: seven patients (19\%) did not answer the telephone, six (17\%) did not remember the contact with the clinical pharmacist, six (17\%) felt too sick to participate, one (3\%) did not want to participate, and one carer (3\%) was not available for interview. Table 2 summarizes the patient characteristics of the 15 participants. The age ranged from 66 to 94 years and there was heterogeneity in the other characteristics as well. All eight hospital wards were represented, and nine clinical pharmacists had been involved in these patients' CMRs. Twelve interviews were held with patients, two with both the patient and carer (in both cases the patients' spouse) and one with the carer only (in this case the patient's daughter).

Table 2 Patient characteristics $(n=15)$

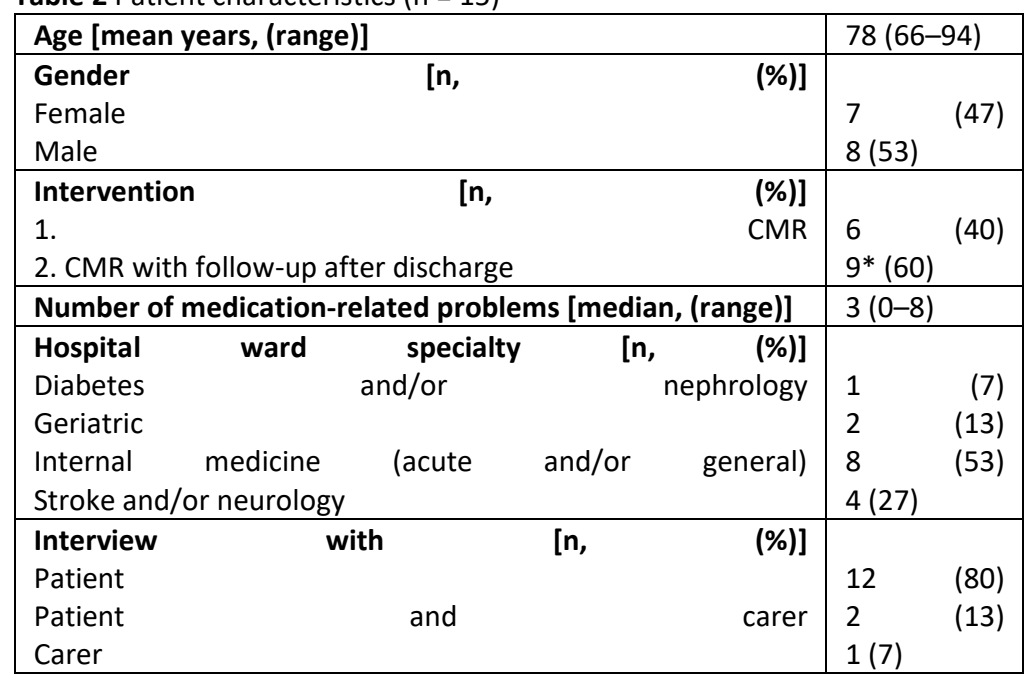

* One patient had already received two follow-up telephone calls when the interview was held.

$\mathrm{CMR}$, comprehensive medication review

\subsection{Key identified themes}

The seven key themes that emerged from the interviews are described below and listed in Table 3 . The findings are supported by illustrative quotes with the interview number in parentheses (P1-14 for the patients; $\mathrm{C6}, \mathrm{C} 9$ and $\mathrm{C} 15$ for the carers).

Table 3 Key identified themes and sub-themes in order of appearance in the text

\begin{tabular}{l} 
Feeling of being taken care of and heterogenous health effects \\
- Patients have a feeling of being taken care of \\
- Understanding of the need of CMRs \\
- Heterogeneous effects on medication treatment \\
- Positive or no effect on treatment adherence \\
\hline The pharmacist is competent* \\
Despite the unclear role of pharmacists, their involvement is appreciated \\
- Unclear role of the pharmacist \\
- Limited understanding of the CMR process \\
- Positive view on pharmacist involvement in healthcare \\
\hline
\end{tabular}




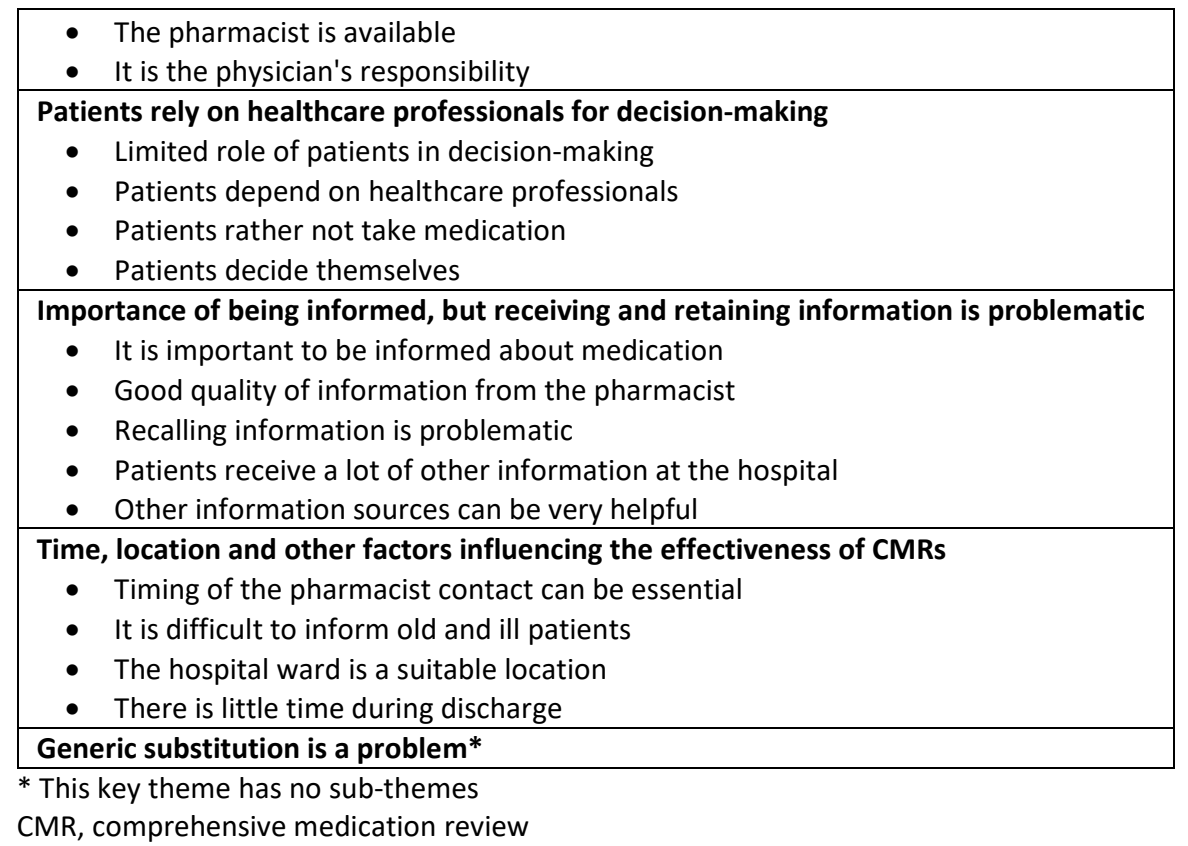

\subsubsection{Feeling of being taken care of and heterogenous health effects}

The contact with the clinical pharmacist, during both the CMRs and follow-up telephone calls, engendered "a feeling of being taken care of" (C6), being listened to and made patients and carers feel "safer" (P8). Patients were generally aware about potential problems with older patients being prescribed multiple medications. They acknowledged the need for CMRs and thought it was good that CMRs were being conducted.

"Elderly get a lot of different medications and eventually it can become a complete mess. Then you have to clean the entire medication list and almost start over." (P12)

For most patients, it was however impossible to say whether the CMRs were effective in terms of health outcomes because they did not exactly know what happened during the CMR process (see paragraph 3.2.3.). Effects on health status, medication treatment or adherence to treatment, that were mentioned by patients were heterogeneous. Some could be "upset" (P2) because medication changes were made which they did not agree with, or they did not notice any difference. Other patients expressed "feeling much better" (P5), or having more control over their medications as a result of the CMR.

\subsubsection{The pharmacist is competent}

Experiences with and views on the pharmacists' competence were only positive. Patients and carers assumed that the pharmacist was "knowledgeable" (P14) about medications, but they also experienced this by getting answers to questions or having discussions about their medications. Patients and carers felt that the pharmacist listened to them, and that the pharmacist was "accommodating" (C9), "calm and collected" (P4). "I could absolutely do that [ask questions about medications], because she was competent." (C15)

\subsubsection{Despite the unclear role of pharmacists, their involvement is appreciated}

Patients and carers knew that the pharmacist checked "whether medications interacted with each other" (P11) and whether the patient received "the right medication" (C15). However, it was unclear what exactly the role of the clinical pharmacist at the ward was, and patients had little understanding about the CMR process.

"I actually did not understand what she [the pharmacist] wanted." (P10)

Several patients were "surprised" (P12) or "confused" (P1) to meet a pharmacist at the ward, because they had "never experienced it before" (P5). They were generally unaware of medication-related problems identified by the pharmacist and discussions between the pharmacist and physician about their medication. 
This could lead to patients being "sceptical" (P4) and not knowing what to ask or expect from the pharmacist. "I sure did have questions, but [...] I did not really know what the conversation was about." (P7)

Despite these issues, pharmacist-physician collaboration in healthcare was generally appreciated.

"You [the pharmacist] with your knowledge and the physicians with theirs can put your heads together and maybe come up with something better than the physician alone." (P4)

Most patients thought it was good that the pharmacist was available at the ward and had the time to go through the patients' medications, because "physicians are very hard to get hold of" (P12). Some patients seemed more indifferent towards the pharmacist being involved. One patient expressed that it was "better to ask a physician" (P1) about medication, because it was the physician's responsibility to prescribe medications.

\subsubsection{Patients rely on healthcare professionals for decision-making}

Patients had a limited role in decision-making during hospital stay: "You don't have that much to say" (P5). They "totally trusted" (P14) healthcare professionals to "do the right thing" (P5), because patients had "no knowledge" (P14) about medication. Most patients just accepted the prescribed medication, even though they "actually didn't want any medication at all" (P8). Some patients mentioned having discussed treatment options with the physician or pharmacist, and thought it would be better if patients were generally more involved.

"I agreed to try it out as she [the pharmacist] suggested." (P7)

"The patient doesn't get involved, but I think it would be very useful to understand why you get this medication." (P13)

Unlike during hospital stay, patients expressed that at home they decided what medication to take. "It is your own choice to refrain from certain medications and take the risk that something happens to you." (P8)

\subsubsection{Importance of being informed, but receiving and retaining information is problematic}

Getting informed about medication was important. Patients expected to receive information about reasons for change and side effects, and to be able to ask questions regarding their medication. This expectation was generally met through the performance of the CMRs and follow-up phone calls, and the information was sufficient and understandable.

"We, the patients, didn't receive so much information before [...] they just gave us our medication and you had to take it without questioning. But now [...] you can question certain things and ask about it, which I think is very important." (P5)

However, information from hospital and primary care could sometimes be conflicting.

"It is quite interesting that one [family] doctor thinks you should take those [medications] and then you're here [at the hospital] and both a pharmacist and a doctor say 'No, she will not have these medications, that is unnecessary'." (P2)

Problems with receiving and retaining information, as part of the CMRs, telephone calls and healthcare in general, were expressed in all interviews. It was difficult to remember who patients had spoken to during hospital stay and what kind of information they had received. Some just had "forgotten everything." (P3) Several patients mentioned having had a good conversation with the pharmacist as part of the CMR, however recalling the content was problematic. This may have been affected by the number of contacts with different healthcare professionals at the hospital.

"Yes, I thought it was a good conversation [...] but unfortunately I cannot be more specific." (P13)

"There were so many doctors whom I talked to, so it's hard to remember." (C9)

Therefore, it was essential for patients to receive written information upon hospital discharge.

"I only received this medication list. They said they had taken away one [medication], but which one, or why, I don't know. [...] I don't like that, I think they should explain why and what could happen. [...] If I wouldn't have received this list, I wouldn't have known which medications I had." (P13) 


\subsubsection{Time, location and other factors influencing the effectiveness of CMRs}

Timing was an important aspect that could influence the perceived effectiveness of the CMRs. The conversation with the pharmacist could be too early, for example if patients were too ill, and the timing of the telephone calls could be unsuitable.

"If you're in hospital like this and just had a stroke, you're not in the right condition [to talk]." (P7) "It was quite a short call, because me and my wife were just on our way out." (P4)

Patients' age and health condition, e.g. "at my age or if you are a little demented" (P2), were mentioned as general factors influencing the performance of CMRs. The hospital ward was a suitable location for a CMR, but lack of time and haste during discharge from the ward were barriers to adequately inform patients.

"We went home after lunch, but it was so messy, and we were in a hurry." (P7)

\subsubsection{Generic substitution is a problem}

Generic substitution, the substitution of a prescribed branded medication by a lower-priced product containing the same active substance [31], was addressed as a serious problem for older patients with regard to their medication management and trust in the effects of their medication, resulting in a high risk for medication errors.

"It is very disturbing for an older person that [...] when you go to the pharmacy to get your medication, the packages look different, the colours of the tablets are different, and the texts are very small. [...] It is definitely a very high risk for a patient to make mistakes." (P10)

\section{Discussion}

\subsection{Discussion of key findings}

The patients' experiences with and views on the CMRs and follow-up telephone calls were generally positive. This positivity was rooted in an understanding of the need for CMRs in older patients using multiple medications, the feeling of being taken care of and listened to, the competences and availability of the wardbased pharmacist, and the provision of understandable and sufficient information to the patients about their medications. These results confirm findings from studies on CMRs by clinical pharmacists in a hospital setting $[22,23]$. Being recognized as an individual, trust in healthcare professionals, communication, information and support are key elements to ensure positive patient experience [28,32], which also seemed to be addressed by the CMRs and follow-up telephone calls in this study.

Physician-pharmacist collaboration was valued, but just as within previous studies [22,23], the unclear or unknown role of the ward-based pharmacist was a barrier to constructive contacts between patients and pharmacists. Patients being surprised, sceptical or not knowing what to expect from the pharmacist, can lead to CMRs not achieving the desired impact on the patients' management of their medications. Informing patients about the presence and role of the pharmacist may improve effectiveness.

Shared decision-making is a key element of patient experience $[28,32]$ and integrated in the CMR process $[8,33,34]$. However, patients expressed a limited role in decision-making during the CMRs within this study. Not all patients wanted to be involved as they relied on the healthcare professionals to provide them with the best care during hospital stay. This is also apparent in other studies where preferences for involvement in decision-making vary substantially, and up to half of the adult patients prefer to passively rely on healthcare professionals rather than playing a more active role $[35,36]$. Higher age and hospital setting, both present in our study, also decrease the willingness to be involved $[37,38]$. In this study, the low level of patient involvement seemed to be related to the lack of understanding about the CMR process. As patients or carers generally did not know whether treatment changes were a result of the CMR or usual care processes at the ward, it was also hard to know the effects of the CMRs.

Despite limited involvement during hospital stay, it was important for patients and carers to be well-informed about their medication treatment upon discharge. However, problems with receiving and retaining information were expressed in all interviews. These problems are common in similar settings, even after receiving structured medication counselling upon discharge [39]. Discharge interventions aiming to increase patient empowerment are effective in reducing hospital readmissions [40]. Information upon or after 
discharge should therefore be tailored to the individual's needs and provided in a way that patients can regain an active role in their medication management $[32,41]$. Although the MedBridge study protocol allowed for patient specific tailoring, the CMR did not necessarily involve patient contact with the pharmacist upon discharge and the pharmacists were not specifically trained in patient empowerment.

Finally, generic substitution was repeatedly mentioned as a problem for medication management by older patients, leading to an increased risk for medication errors. Despite this being a well-known issue [31,42], it was not specifically addressed within the CMRs, as the ward-based pharmacists were not aware of which generic medications were dispensed at the community pharmacy. Closer collaboration between stakeholders on a national level may provide a solution to this problem.

\subsection{Limitations}

Despite multiple strategies to ensure trustworthiness and rigour of the findings, limitations exist. Patients were excluded for interview if they were not taking care of their own medication management at home, and one-sixth of all patients who were approached did not specifically remember the contact with the clinical pharmacist. This limits the credibility of our findings, but it also supports the key themes on the unclear role of pharmacist and problems with recalling information. The researcher who conducted the interviews had a background in pharmacy, which may have limited the honesty of the participants. To mitigate this limitation, confidentiality was emphasized and participants were encouraged to be frank and talk freely. Both researchers involved in the analysis were involved as study coordinators in the MedBridge trial, which may have led to coloured interpretations of the findings, consequently lowering confirmability. We therefore provided quotes from all participants to substantiate the identified themes. Finally, the transferability of the findings to other contexts may be limited, as we only included participants from four hospitals in Sweden. However, our results confirm findings from studies on similar initiatives within other countries and healthcare settings.

\subsection{Conclusion}

Older patients generally have positive experiences with and views on CMRs and follow-up telephone calls by ward-based clinical pharmacists. However, some factors, like the unclear role of the ward-based pharmacist and problems with receiving and retaining information, may negatively impact the effectiveness of these interventions.

\subsection{Practice implications}

This study supports the understanding of the effects of hospital-initiated CMRs and follow-up by clinical pharmacists on patients' health outcomes within the MedBridge trial. Future initiatives on similar interventions should utilize older patients' acknowledgement of the need for CMRs, trust in the competence of the pharmacist and appreciation of physician-pharmacist collaboration. These findings also raise questions for future research and practice, such as how to promote an understanding of the presence and role of the pharmacist at the ward, optimise patient communication and improve effectiveness of the CMRs. Instead of involving all hospitalized patients in decision-making, it seems essential to focus on enabling the patient to regain the role of active participant after discharge, understanding the results of the CMR process and changes to medication treatment. Future initiatives should also find out how to address problems with generic substitution to ensure optimal medication management by older patients.

\section{Author contributions}

TK: Funding Acquisition, Conceptualization, Methodology, Formal analysis and writing (original draft), Writing (review and editing)

AK: Methodology, Formal analysis and writing (original draft), Writing (review and editing)

UG: Funding Acquisition, Conceptualization, Methodology, Writing (review and editing), Supervision

DS: Conceptualization, Methodology, Writing (review and editing), Supervision 


\section{Conflicts of interest}

None.

\section{Acknowledgements}

The authors are thankful to all participating patients within this study. This study is part of the MedBridge project which has received governmental research funding from the Uppsala-Örebro Regional Research Council [grant RFR-555601, RFR-641791 and RFR-735911], Region Uppsala [grant LUL-527721, LUL-614061 and LUL-716201], Region Gävleborg [grant CFUG-658451 and CFUG-698771] and Region Västmanland [grant LTV-675921, LTV-712341 and LTV-736641]. The project has also received funding from the Swedish Pharmacists Association [Sveriges Farmaceuter], the Thuréus Fund for Geriatric Research [Thuréus stiftelse för främjande av geriatrisk forskning], the Geriatric Fund [Geriatriska fonden] and the Swedish Heart and Lung Association [Riksförbundet HjärtLung; grant FA 2017:38]. No sponsor had any involvement in the study design, in the collection, analysis or interpretation of data, in the writing of the report, or in the decision to submit the article for publication.

\section{References}

1. Chiatti C, Bustacchini S, Furneri G, Mantovani L, Cristiani M, Misuraca C, et al. The Economic Burden of Inappropriate Drug Prescribing, Lack of Adherence and Compliance, Adverse Drug Events in Older People. Drug Saf. 2012;35:73-87.

2. World Health Organisation. WHO Global Patient Safety Challenge: Medication Without Harm [Internet]. 2017 [cited $2018 \mathrm{Jul}$ 19]. Available from: http://www.who.int/patientsafety/medicationsafety/medication-without-harm-brochure/en/

3. Leendertse AJ, Visser D, Egberts ACG, van den Bemt PMLA. The relationship between study characteristics and the prevalence of medication-related hospitalizations: a literature review and novel analysis. Drug Saf. 2010;33:233-44.

4. McLachlan CYL, Yi M, Ling A, Jardine DL. Adverse drug events are a major cause of acute medical admission. Intern Med J. 2014;44:633-8.

5. Winterstein AG, Sauer BC, Hepler CD, Poole C, Suárez EC, Kaiser JM. Preventable drug-related hospital admissions. Ann Pharmacother. 2002;36:1238-48.

6. Garcia-Caballos M, Ramos-Diaz F, Jimenez-Moleon JJ, Bueno-Cavanillas A. Drug-related problems in older people after hospital discharge and interventions to reduce them. Age Ageing. 2010;39:430-8.

7. Knight DA, Thompson D, Mathie E, Dickinson A. 'Seamless care? Just a list would have helped!' Older people and their carer's experiences of support with medication on discharge home from hospital. Heal Expect. 2013;16:277-91.

8. NICE Medicines and Prescribing Centre (UK). Medicines Optimisation: The Safe and Effective Use of Medicines to Enable the Best Possible Outcomes [Internet]. Manchester: National Institute for Health and Care Excellence (UK); 2015. Available from: https://www.nice.org.uk/guidance/ng5

9. Blenkinsopp A, Bond C, Raynor DK. Medication reviews. Br J Clin Pharmacol. 2012;74:573-80.

10. Gillespie U, Alassaad A, Henrohn D, Garmo H, Hammarlund-Udenaes $M$, Toss $H$, et al. A Comprehensive Pharmacist Intervention to Reduce Morbidity in Patients 80 Years or Older: A Randomized Controlled Trial. Arch Intern Med. 2009;169:894-900.

11. Christensen $M$, Lundh $A$. Medication review in hospitalised patients to reduce morbidity and mortality. Cochrane Database Syst Rev. 2016;CD008986.

12. Kempen TGH, Bertilsson M, Lindner K-J, Sulku J, Nielsen EI, Högberg A, et al. Medication Reviews Bridging Healthcare (MedBridge): Study protocol for a pragmatic cluster-randomised crossover trial. Contemp Clin Trials. 2017 Oct;61:126-32.

13. Moore G, Audrey S, Barker M, Bonell C, Hardeman W, Moore L, et al. Process evaluation of complex interventions. UK Medical Research Council (MRC) guidance. 2014.

14. Doyle C, Lennox L, Bell D. A systematic review of evidence on the links between patient experience and clinical safety and effectiveness. BMJ Open. 2013;3:e001570.

15. Jenkinson C, Coulter A, Bruster S. The Picker Patient Experience Questionnaire: development and validation using data from in-patient surveys in five countries. Int J Qual Heal Care. 2002;14:353-8. 
16. Ruiz-Millo O, Climente-Marti M, Navarro-Sanz JR. Patient and health professional satisfaction with an interdisciplinary patient safety program. Int J Clin Pharm. 2018;40:635-41.

17. Kim S, Martin MT, Pierce AL, Zueger P. Satisfaction With Medication Therapy Management Services at a University Ambulatory Care Clinic. J Pharm Pract. 2016;29:199-205.

18. Christy S, Sin B, Gim S. Impact of an Integrated Pharmacy Transitions of Care Pilot Program in an Urban Hospital. J Pharm Pract. 2016;29:490-4.

19. Creswell JW. Designing a Qualitative Study. In: Qualitative inquiry and research design: choosing among five approaches. 3rd ed. Thousand Oaks, California, USA: SAGE Publications, Inc; 2013. p. 448.

20. Usher-Smith JA, Harte E, MacLure C, Martin A, Saunders CL, Meads C, et al. Patient experience of NHS health checks: a systematic review and qualitative synthesis. BMJ Open. 2017;7:e017169.

21. Gibbons C, Singh S, Gibbons B, Clark C, Torres J, Cheng MY, et al. Using qualitative methods to understand factors contributing to patient satisfaction among dermatology patients: a systematic review. J Dermatolog Treat. 2017;11:1-5.

22. Garcia B, Storli S, Småbrekke L. A pharmacist-led follow-up program for patients with coronary heart disease in North Norway-a qualitative study exploring patient experiences. BMC Res Notes. 2014;7:197.

23. Morecroft CW, Thornton D, Caldwell NA. Inpatients' expectations and experiences of hospital pharmacy services: qualitative study. Heal Expect. 2015;18:1009-17.

24. Ritchie J, Lewis J. Qualitative research practice : a guide for social science students and researchers. Sage Publications; 2003. 336 p.

25. O'Brien BC, Harris IB, Beckman TJ, Reed DA, Cook DA. Standards for Reporting Qualitative Research. Acad Med. 2014;89:1245-51.

26. Tong A, Sainsbury P, Craig J. Consolidated criteria for reporting qualitative research (COREQ): a 32item checklist for interviews and focus groups. Int J Qual Heal Care. 2007;19:349-57.

27. Francis JJ, Johnston M, Robertson C, Glidewell L, Entwistle V, Eccles MP, et al. What is an adequate sample size? Operationalising data saturation for theory-based interview studies. Psychol Heal. 2010;25:1229-45.

28. UK Department of Health. NHS Patient Experience Framework [Internet]. 2012 [cited 2017 Sep 17]. Available from: https://www.gov.uk/government/publications/nhs-patient-experience-framework

29. ICT Services and System Development and Division of Epidemiology and Global Health. Open Code 4.03 [Internet]. Umeå: Umeå Unversity; 2013. Available from: http://www.phmed.umu.se/english/units/epidemiology/research/open-code/

30. Shenton AK. Strategies for ensuring trustworthiness in qualitative research projects. Educ Inf. 2004;22:63-75.

31. Posner J, Griffin JP. Generic substitution. Br J Clin Pharmacol. 2011;72:731-2.

32. Staniszewska S, Boardman F, Gunn L, Roberts J, Clay D, Seers K, et al. The Warwick Patient Experiences Framework: patient-based evidence in clinical guidelines. Int J Qual Heal Care. 2014;26:151-7.

33. Socialstyrelsen. Medication reviews for elderly prescribed five or more drugs [Läkemedelsgenomgångar för äldre ordinerade fem eller fler läkemedel]. 2013th-3rd-18th ed. Stockholm, Sweden: National Board of Health and Welfare; 2013. 32 p.

34. American Pharmacists Association, National Association of Chain Drug Stores Foundation. Medication Therapy Management in Pharmacy Practice: Core Elements of an MTM Service Model Version 2.0 [Internet]. Available from: https://www.pharmacist.com/

35. Levinson W, Kao A, Kuby A, Thisted RA. Not all patients want to participate in decision making. A national study of public preferences. J Gen Intern Med. 2005;20:531-5.

36. Kayyali R, Gebara SN, Hesso I, Funnell G, Naik M, Mason T, et al. Shared decision making and experiences of patients with long-term conditions: has anything changed? BMC Health Serv Res. 2018;18:763.

37. Shay LA, Lafata JE. Understanding patient perceptions of shared decision making. Patient Educ Couns. 2014;96:295-301.

38. Street RLJ, Gordon HS, Ward MM, Krupat E, Kravitz RL. Patient participation in medical consultations: why some patients are more involved than others. Med Care. 2005;43:960-9.

39. Eibergen L, Janssen MJA, Blom L, Karapinar-Çarkit F. Informational needs and recall of in-hospital 
medication changes of recently discharged patients. Res Soc Adm Pharm. 2018;14:146-52.

40. Braet A, Weltens $C$, Sermeus W. Effectiveness of discharge interventions from hospital to home on hospital readmissions: a systematic review. JBI database Syst Rev Implement reports. 2016;14:10673.

41. Borgsteede SD, Karapinar-Carkit F, Hoffmann E, Zoer J, van den Bemt PMLA. Information needs about medication according to patients discharged from a general hospital. Patient Educ Couns. 2011;83:228.

42. Dunne SS. What Do Users of Generic Medicines Think of Them? A Systematic Review of Consumers' and Patients' Perceptions of, and Experiences with, Generic Medicines. Patient. 2016;9:499-510. 